\title{
IN-DROPLET PARTICLE SEPARATION BY TRAVELLING WAVE DIELECTROPHORESIS (twDEP) AND EWOD
}

\author{
Yuejun Zhao ${ }^{\mathrm{a}}$, Ui-Chong $\mathrm{Yi}^{\mathrm{b}}$, and Sung Kwon Cho ${ }^{\mathrm{a}^{*}}$ \\ ${ }^{a}$ Department of Mechanical Engineering, University of Pittsburgh \\ Pittsburgh, PA 15261, USA \\ ${ }^{\mathrm{b}}$ Core Microsolutions Inc. \\ Los Angeles, CA 90024, USA
}

\begin{abstract}
This paper describes successful particle separations within a droplet by traveling wave dielectrophoresis (twDEP) and consecutive droplet splitting by electrowetting-on-dielectric (EWOD). This in-droplet method will provide a new functionality of separation and concentration for digital (droplet-based) microfluidics [1], thereby possibly eliminating the necessity of adopting the conventional microchannel-based separation methods.
\end{abstract}

\section{INTRODUCTION}

There are currently high demands for means to efficiently manipulate and discriminate bio entities in microfluidics $[2,3]$. To date, numerous systems have been developed to meet these demands. However, most of them are based on continuous flows which basically require complicated micro channel networks, consequently raising the cost of design and fabrication and lowering the reliability of operation. Recently, in the mean time, digital (droplet-based) microfluidics [1] (in particular operated by electrowetting-on-dielectric (EWOD)) successfully demonstrated various fluidic operations, obviating the above drawbacks. A large number of individual minute droplets carrying bio samples can be manipulated (created, transported, split, and mixed) in parallel on a two-dimensional (2-D) surface, with no need to build any micro channel structures. Furthermore, all of these operations can be accomplished under digital control from a programmable controller, promising tremendous potential for chemical and biologic analysis [4].

However, the implementation of bio sample concentration and separation in digital microfluidics is still challenging since most of the previously developed concentration and separation methods essentially rely on long micro channels (e.g. micro capillary electrophoresis). Therefore, simply adopting the conventional concentration and separation methods into digital microfluidics requires constructing microchannel networks, as a result deteriorating the original strength of digital microfluidics. Most recently, with a motivation to realize concentration and separation in digital microfluidics without microchannels, Cho and Kim [5] presented an in-droplet particle separation and concentration control by using electrophoresis in a droplet configuration. However, this technique exclusively works only with electrically charged particles, limiting its application for non or weakly charged particles.

On the contrary, dielectrophoresis (DEP) [6] accesses a wide range of particle characteristics by changing the frequency of the applied electric field and by using different suspending media. In DEP particle separations, particles to be separated need no charge treatments. In particular, traveling wave dielectrophoresis (twDEP), originally described by Masuda et al $[7,8]$, that uses a traveling electric field with numerous electrodes allows long distance transportations of various particles. Therefore, this principle has currently been developed into a comprehensive technique for manipulating and separating various particles and cells [9-12]. To our knowledge, however, dielectrophoretic particle separations have rarely or never been successfully implemented in digital (droplet-based) microfluidics. Hence, in this paper, we implement twDEP particle separations within a droplet and furthermore integrate them with follow-up EWOD droplet splitting, thereby eliminating any possibility of particle dispersion posterior to separation.

\section{CONCEPT}

Figure 1 illustrates sequential procedures of the proposed indroplet particle separation: (1) initial state with a droplet containing mixed particles (Fig. 1(a)); (2) transportation of target particles (shown as black) into the right edge of the droplet by twDEP (Fig. 1(b)); (3) droplet splitting by EWOD (Fig. 1(c)); (4) final state (Fig. 1(d)). For twDEP particle separation, four spiral electrodes (Fig. 4(a)) placed on the bottom plate are used to generate a traveling electric field. Then they are switched for grounding when EWOD is on for droplet splitting (Fig. 1(c)). Meanwhile, three transparent electrodes placed on the top plate are used for the EWOD droplet splitting. The left and right electrodes (except the middle one) are activated to elongate and split the droplet (Fig. 1(c)). For improved separation precision, multiple droplets with the same particle type can be re-joined and may go through further separation in the next stage.

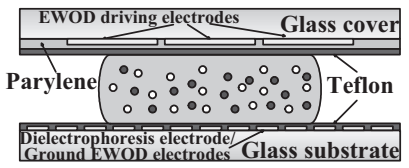

(a)

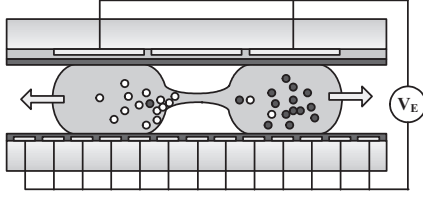

(c)

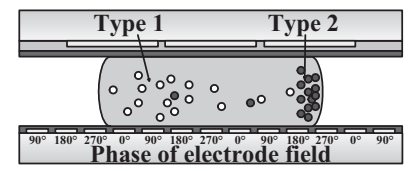

(b)

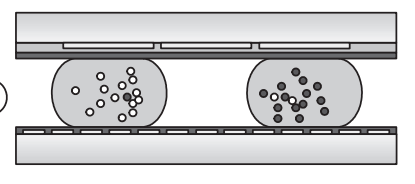

(d)
Figure 1. The schematic of particle separation procedures in a droplet: (a) A droplet containing mixed particles is placed between two parallel plates; (b) under the traveling wave dielectrophoresis (twDEP) generated by the bottom electrodes, target particles (type 2, black) are transported and concentrated on the right side; (c) under the electrowetting-on-dielectric (EWOD) force generated by the top electrodes, the droplet is split into two daughter droplets; (d) finally two types of particles are separated into respective two daughter droplets.

\section{FABRICATION OF TESTING DEVICES}

The testing devices that mainly consist of two parallel plates (top and bottom) were fabricated using standard lithographic micro 
fabrication technology. The bottom plate fabrication includes two steps: metallization and patterning of twDEP electrodes and deposition of the hydrophobic layer. For the twDEP electrodes, a

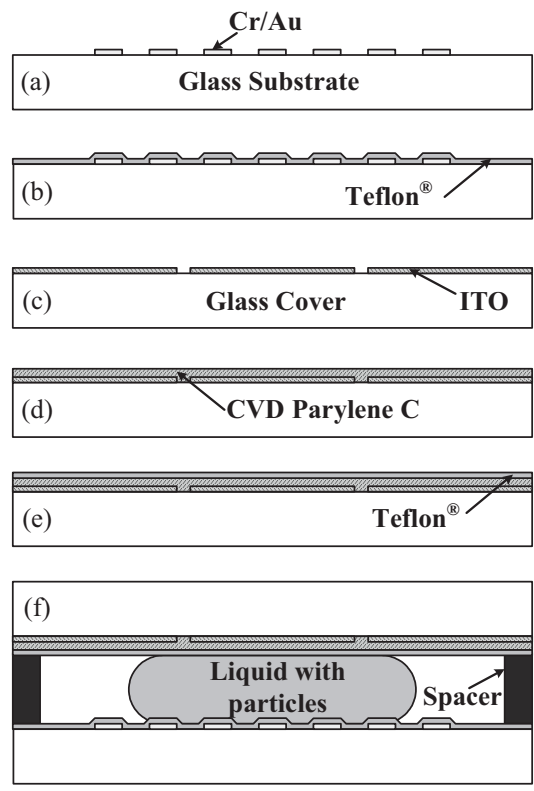

Figure 2. Fabrication process flow of testing devices: (a) deposition and patterning of electrodes $(\mathrm{Cr} / \mathrm{Au})$ on bottom glass; (b) spin-coating of Teflon ${ }^{\mathbb{B}}$ AF; (c) Top glass cover with patterned ITO layer; (d) Deposition of parylene layer by CVD and patterning; (e) Spin-coating of Teflon ${ }^{\circledR}$ AF layer; (f) Putting the droplet containing particles and integration of the top and bottom plates with a spacer in between.

chromium layer of $100 \AA$ in thickness as an adhesion layer and a gold layer of $1000 \AA$ in thickness were sequentially deposited on a glass wafer by sputtering and then patterned by wet etching into parallel electrodes of $10 \mu \mathrm{m}$ width and $10 \mu \mathrm{m}$ spacing. The bottom plate was finally coated with a hydrophobic Teflon layer. Spincoating of $2 \%$ Teflon solution (Teflon AF $1600^{\circledR}+$ Fluorocarbon solvent) resulted in a $2000 \AA$ thick Teflon layer. The main fabrication process on the top plate consists of three steps: patterning of EWOD electrodes, deposition and patterning of the dielectric layer, and deposition of the hydrophobic layer. For the driving electrodes, an ITO (Indium Tin Oxide) layer was patterned by wet etching. Then, a $2 \mu \mathrm{m}$ parylene layer as a dielectric layer was deposited using a vapor deposition method (Specialty Coating Systems: PDS-2010 LABCOTER $^{\circledR} 2$ ), followed by opening of the parylene layer on electrode pads by physical scratching in order to transmit activation signals to each driving electrode. Finally, the top plate was also spin-coated with the hydrophobic Teflon layer.

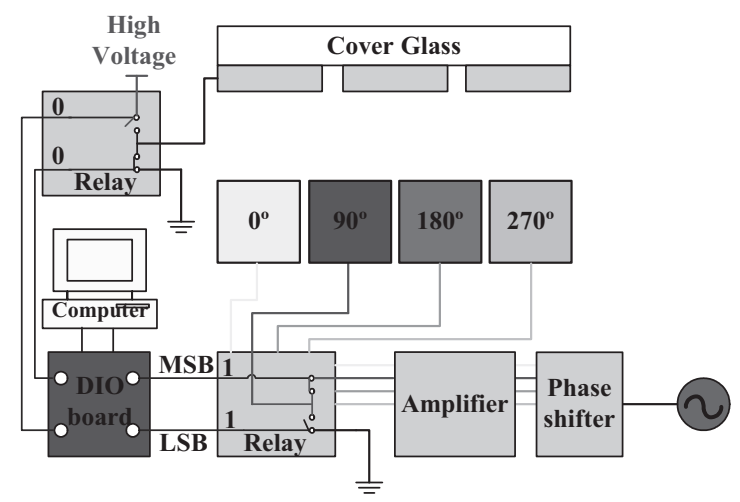

Figure 3. Schematic of activation signal flows.
The last step of the fabrication process is to integrate the two plates (Fig. 2(f)). After putting a droplet containing particles on the bottom plate, the top cover plate was gently pressed against the spacers that were already placed on the bottom plate. The clean room tape was used for the spacers, making the channel gap at 70 $\mu \mathrm{m}$ that allows successful droplet splitting based on the design rule [1].

Figure 3 illustrates how to generate EWOD/twDEP signals. The whole system consists of a personal computer, a digital output board (DAQPad-6507, National Instrument), a custom-made phase shifter, amplifier and interface circuit mainly containing photocoupled relays $\left(\right.$ PhotoMos $^{\circledR}$, AQW614EH, Aromat Co.). A PCbased program generates control signals transmitted through a digital I/O board. The control signals switch the relays through which a $90^{\circ}$ shift traveling wave is transmitted to the bottom plate electrodes and EWOD activation voltages to the top plate electrodes on the testing devices.

\section{EXPERIMENT RESULTS}

As a first step, we tested particle transportation and concentration in a droplet using a single type of particle $(5-\mu \mathrm{m}$ aldehyde sulfate (AS) beads). A traveling wave of $90^{\circ}$ phase shift to the adjacent electrodes is generated with a four spiral electrodes design. To avoid multi-layer wiring, a spiral type was used, as shown in Fig. 4(a). Note that the droplet is largely placed on the area of parallel electrodes, not touching the center of the spiral electrodes. Before twDEP actuation, 5 - $\mu \mathrm{m}$ aldehyde sulfate (AS) beads (seen as white dots) are initially uniformly suspended in the droplet; under the twDEP wave $(10 \mathrm{kHz}, 10 \mathrm{Vp}-\mathrm{p})$ almost all the particles move to the right region of the droplet (Figs. 4(b) and (c)).

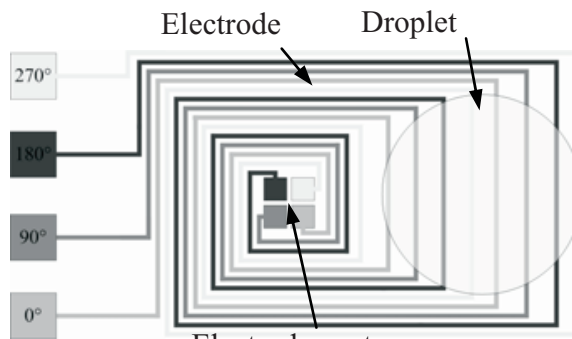

Electrode center

(a)

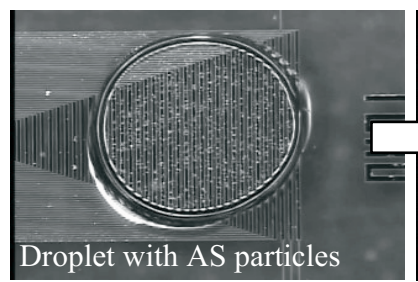

(b)

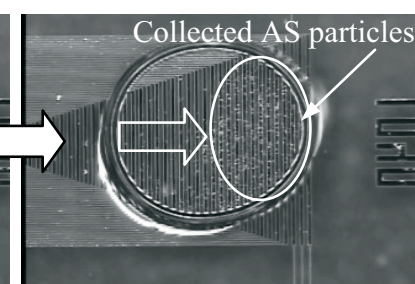

(c)
Figure 4. Top views of transportation of particle of single type into the right side of the droplet: (a) Electrodes design; (b) Before twDEP actuation, $5 \mu \mathrm{m}$ aldehyde sulfate $(A S)$ beads are uniformly suspended in the droplet; (c) Almost all the particles move to the right region of the droplet under the twDEP wave $(10 \mathrm{kHz}, 10 \mathrm{Vp}$ p).

Next, twDEP particle separations are performed in the following three different mixture cases: (case 1, Fig. 5) 8- $\mu \mathrm{m}$ glass beads and 5- $\mu \mathrm{m}$ AS beads; (case 2, Fig. 6) 8- $\mu \mathrm{m}$ glass beads and ground pine spores (GPS); (case 3, Fig. 7) 5- $\mu \mathrm{m}$ AS beads and 10$\mu \mathrm{m}$ CML beads. 


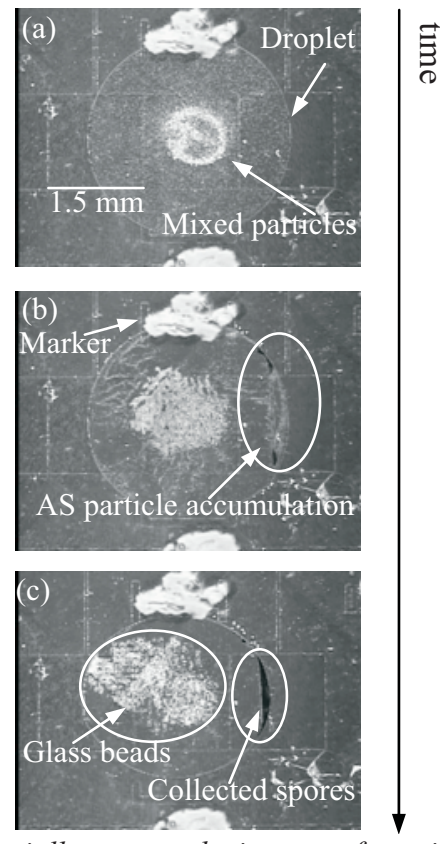

Figure 5. Sequentially captured pictures of particle separation

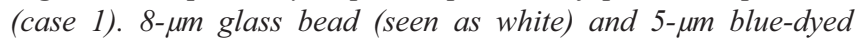
aldehyde sulfate (AS) beads (seen as black) are initially mixed (a) and then separated under a twDEP wave of $800 \mathrm{kHz}$ and $15 \mathrm{Vp}-\mathrm{p}$ $(b, c)$.

Figure 5 shows the sequentially captured pictures of particle separation for case 1 . Initially 8 - $\mu \mathrm{m}$ glass beads (seen as white) and $5-\mu \mathrm{m}$ blue-dyed aldehyde sulfate (AS) beads (seen as black) are mixed in the droplet as shown in Fig. 5(a). The AS particles are moving to the right edge of the droplet under $800 \mathrm{kHz}$ and $15 \mathrm{Vp}-\mathrm{p}$ while the glass beads are circulating and overall a little bit pushed

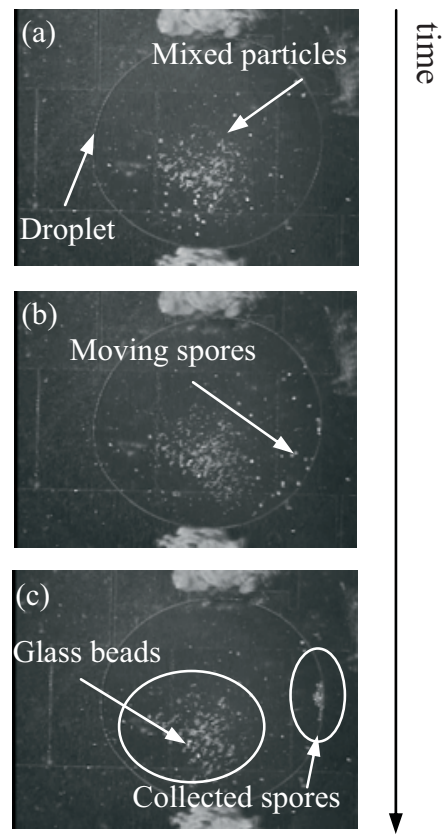

Figure 6. Sequentially captured pictures of particle separation (case 2). Lycopodium powder (Ground Pine Spores or GPS seen as big white dots) and glass beads (seen as small white dots) are mixed (a) and then separated under a traveling wave of $800 \mathrm{kHz}$ and $20 \mathrm{Vp}-\mathrm{p}(b, c)$. to the left; After several minutes almost all the AS (seen as black) particles are collected in the right edge of the droplet as shown in Figs. 5 (b) and (c). Note that the white makers were intentionally placed to help to position the droplet.

Figure 6 shows the sequential pictures of spore separation for case 2. Similar to case 1, successful separation was achieved. Before separation, Lycopodium powder (Ground Pine Spores or GPS seen as big white dots) and glass beads (seen as small white dots) are mixed in the droplet as shown in Fig. 6(a). The spores are moved to the right under $800 \mathrm{kHz}$ and $20 \mathrm{Vp}$-p while the glass beads are a little bit pushed to the left; After several minutes the spores are collected at the right edge of the droplet as shown in Figs. 6 (b) and (c).
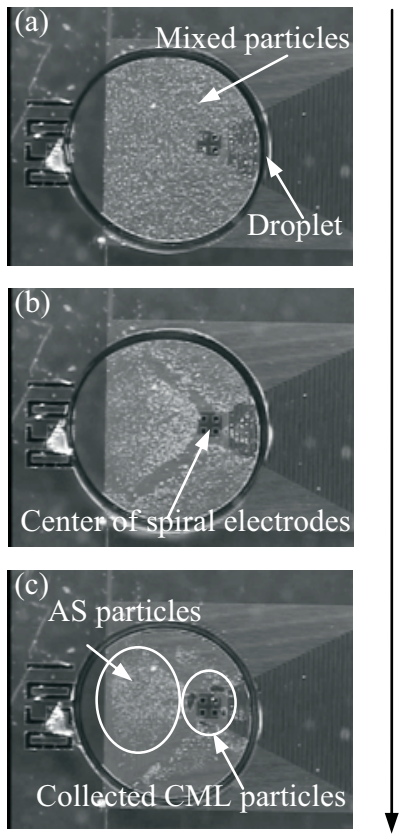

Figure 7. Sequentially captured pictures of particle separation (case 3). 5- $\mu m$ aldehyde sulfate (AS) beads (seen as white) and 10um CML (seen as black) particles are mixed.

Figure 7 shows the sequentially captured pictures of particle separation for case 3 . Initially 5 - $\mu \mathrm{m}$ aldehyde sulfate (AS) beads (seen as white) and $10-\mu \mathrm{m}$ CML (seen as black) particles are mixed in the droplet as shown in Fig. 7(a). The CML (black) particles are moving to right region (the center of the spiral electrodes) under $50 \mathrm{kHz}$ and $4 \mathrm{Vp}-\mathrm{p}$ while the AS beads (white) are circulating as shown in Fig. 7(b). After several minutes CML (black) particles are collected in the right area while the AS beads (white) suspends in the left region as shown in Fig. 7(c). Note that the droplet is positioned such that the center of the spiral electrodes is placed inside the droplet contour, differently from the cases in Figs. 4, 5, and 6. As a consequence, the CML beads (black) are collected near the center of the spiral electrodes while cases 1 and 2 show target particle collections in the right edge of the droplet since the droplet largely covers the area of parallel electrode pattern (not the center of the electrodes). This means that the target particle collection region can be controllably positioned within a droplet depending on where the droplet is placed on the spiral electrodes.

Lastly, figure 8 shows particle separation and subsequent droplet splitting. Initially $8-\mu \mathrm{m}$ glass beads (seen as big white dots) and 5 - $\mu \mathrm{m}$ blue-dyed aldehyde sulfate (AS) beads are mixed in the mother droplet as shown in Fig. 8(a). AS particles (seen as small 
white dots) are moving to the right side of the droplet under 800 $\mathrm{kHz}$ and $15 \mathrm{Vp}-\mathrm{p}$ while the glass beads are circulating and overall a little bit pushed to the left as shown in Fig. 8(b). Splitting function by EWOD (1 kHz and $150 \mathrm{Vp}-\mathrm{p})$ makes the droplet physically divided into two daughter droplets. After splitting, the left droplet is glass beads rich while the right one is AS beads rich as shown in Figs. 8(c) and (d). This splitting step enables to eliminate particle dispersion problems after completing the separation.
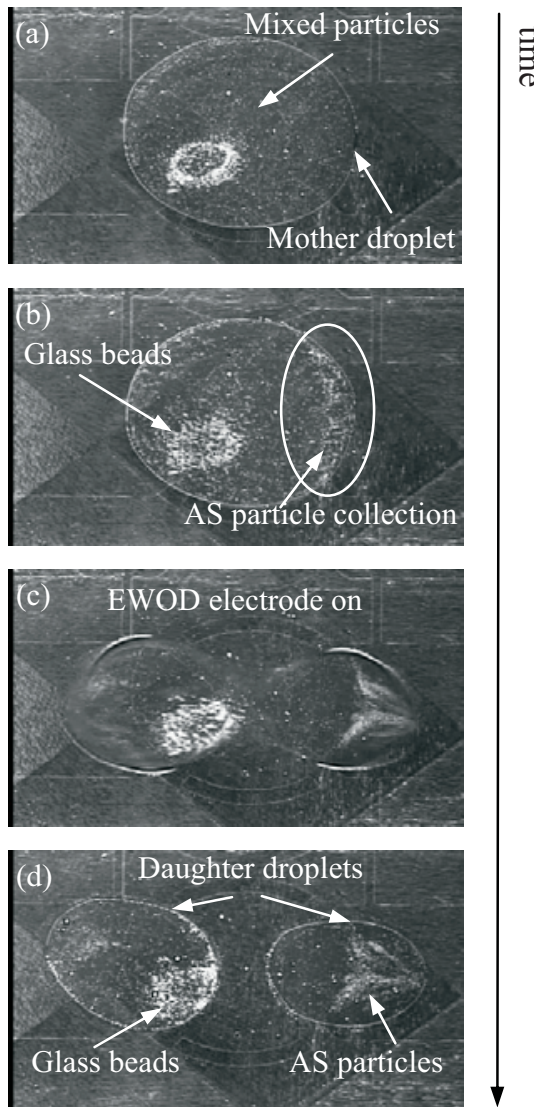

Figure 8. Sequentially captured pictures of particle separation and droplet splitting. 8-um glass beads (seen as big white dots) and 5-um blue-dyed aldehyde sulfate (AS) beads (seen as small white dots) are mixed, then separated by twDEP (800 kHz and 15 $V p-p)$, and finally secured in each daughter droplet produced by EWOD splitting process.

\section{CONCLUSIONS}

This paper describes successful twDEP (traveling wave dielectrophoresis) particle separations in droplet configurations and subsequent droplet splitting. 5 different cases of particle combinations have been examined and their successful concentration and separation have been experimentally demonstrated. Furthermore, subsequent EWOD droplet splitting was successfully integrated, securing separated particles in each produced daughter droplet. This splitting step enables to essentially avoid particle dispersion problems that otherwise would deteriorate the separation efficiency posterior to the separation. This integrated in-droplet separation method will provide a new functionality of separation and concentration for digital (dropletbased) microfluidics, thereby possibly eliminating the necessity of adopting the conventional microchannel-based separation methods.

\section{REFERENCES}

[1] S. K. Cho, H. Moon, and C.-J. Kim, "Creating, Transporting, Cutting and Merging of Liquid Droplets by Electrowetting-Based Actuation for Digital Microfluidic Circuits," Journal of Microelectromechanical Systems, 12, 1, pp. 70 - 80 (2003).

[2] P. R. C. Gascoyne, and J. V. Vykoukal, "Particle Separation by Dielectrophoresis," Electrophoresis, 23, pp. 1973 - 1983 (2002).

[3] C. H. Kua, Y. C. Lam, C. Yang, and K. Youcef-Toumi, "Review of Bio-particle Manipulation Using Dielectrophoresis," Innovation in Manufacturing Systems and Technology, (2005). http://hdl.handle.net/1721.1/7464

[4] P. R. C. Gascoyne, and J. V. Vykoukal, "DielectrophoresisBased Sample Handling in General-Purpose Programmable Diagnostic Instruments," Proceedings of the IEEE, 92, 1, pp. 22 42 (2004).

[5] S. K. Cho, and C.-J. Kim, "Particle Separation and Concentration Control for Digital Microfluidics Systems", MEMS 2003 Conference, Kyoto, Japan (2003), pp. 686 - 689.

[6] H. A. Pohl, Dielectrophoresis: The behavior of neutral matter in nonuniform electric fields, Cambridge University Press, Cambridge, New York (1978).

[7] S. Masuda, M. Washizu, and M. Iwadare, "Separation of Small Particles Suspended in Liquid by Non-uniform Travelling Field," IEEE Transactions on Industry Applications, 23, pp. 474 - 480 (1987).

[8] S. Masuda, M. Washizu, and I. Kawabata, "Movement of Blood Cells in Liquid by Non-uniform Travelling Field," IEEE Transactions on Industry Applications, 24, pp. 217 - 222 (1988).

[9] M. P. Hughes, R. Pethig, and X. B. Wang, "Dielectrophoretic Forces on Particles in Travelling Electric Fields," Journal of Physics D: Applied Physics, 29, pp. 474 - 482 (1996).

[10] H. Morgan, N. G. Green, M. P. Hughes, W. Monaghan, and T. C. Tan, "Large-area Travelling-wave Dielectrophoresis Particle Separator," Journal of Micromechanics and Microengineering, 7, pp. 65 - 70 (1997).

[11] P. R. C. Gascoyne, C. Mahidol, M. Ruchirawat, J. Satayavivad, P. Watcharasit, and F. F. Becker, "Microsample Preparation by Dielectrophoresis_Isolation of Malaria," Lab Chip, 2, pp. 70 - 75 (2002).

[12] L. M. Fu, G. B. Lee, Y. H. Lin, and R. J. Yang, "Manipulation of Microparticles Using New Modes of TravelingWave-Dielectrophoretic Forces: Numerical Simulation and Experiments," IEEE /ASME Transactions on Mechatronics, 9, 2, pp. 377 - 383 (2004). 